\title{
A REVIEW ON VARIOUS ROUTING PROTOCOLS FOR MANET
}

\author{
D.Bhadru ${ }^{1}$ and Venkateswarulu Naik.B ${ }^{2}$
}

\begin{abstract}
Mobile Ad-hoc Network (MANET) is a very powerful field in the progress of Wireless network. Over the past years Wireless ad-hoc network become most stimulating and vital field due to the popularity of mobile devices and wireless network. MANET is a network with many autonomous nodes i.e. mobile devices. MANET uses dynamic topology, wireless links, decentralized network and doesn't need any cellular infrastructure. Due to mobile nature of nodes, the network topology may change rapidly and unpredictably time to time The network is decentralized, where all network activity including discovering the topology and delivering messages must be executed by the nodes themselves, i.e., routing functionality will be incorporated into mobile nodes. Many routing algorithms have been proposed and developed for accomplishing this task. Therefore, it is difficult to determine, in this paper we investigate Desirable Properties of Ad-Hoc Routing Protocolsperforms best under different situations like multiple routes, Routing Philosophy, Routing Schemes, Routing Overheads, latency, Scalability or Reliability, congestion, Security.
\end{abstract}

Keywords: MANET, AODV, DSR, TORA, Proactive, Reactive and Hybrid protocol

\section{INTRODUCTION}

Mobile Ad-hoc network (MANET) becomes one of the most capable fields for research. MANET is a wireless adhoc network it can be connected to internet or external network and can be a standalone network. A mobile ad hoc network (MANET) is a kind of wireless network without centralized administration or fixed network infrastructure in which nodes communicate over relatively bandwidth constrained wireless links and perform routing discovery and routing maintenance in a self-organized way. Due to mobile nature of nodes, the network topology may change rapidly and unpredictably time to time. The network is decentralized, where all network activity including discovering the topology and delivering messages must be executed by the nodes themselves, i.e. routing functionality will be incorporated into mobile nodes. Due to these reasons routing in MANETs is a challenging task. Multicast plays an important role in MANET. Nowadays the MANET enables many applications in the areas of emergency operations, disaster relief efforts. The Mobile Ad hoc network is one of most commonly used wireless network. As the number of user increases MANET suffer from most common network problems like Factors such as variable wireless link quality, propagation path loss, fading, multi-user interference, power expended, and topological changes, become relevant issues. The network should be able to adaptively alter the routing paths to alleviate any of these effects. Moreover, in a military environment, preservation of security, latency, reliability, intentional jamming, and recovery

\footnotetext{
${ }^{1}$ Assistant Professor SVIT, Secundrabad

${ }^{2}$ Assistant Professor ELCET, Hyderabad
} 
from failure are significant concerns. Military networks are designed to maintain a low probability of intercept and/or a low probability of detection. Hence, nodes prefer to radiate as little power as necessary and transmit as infrequently as possible, thus decreasing the probability of detection or interception. A lapse in any of these requirements may degrade the performance and dependability of the network.

\section{DESIRABLE PROPERTIES OF AD-HOC ROUTING PROTOCOLS}

In the design of routing protocols for mobile ad-hoc networks, the following are desirable factors: -

(1) Distributed operation: - With no central hierarchy of routers, routing must be distributed amongst the participant nodes.

(2) Loop-freedom: - Aim to avoid route discovery or maintenance processes from spinning from node to node indefinitely.

(3) Demand-based operation versus Proactive operation: - To minimize the control overhead in the network and thus not waste the network resources the protocol should be reactive. This means that the protocol should react only when needed and that the protocol should not periodically broadcast control information.

(4)Unidirectional link support: The radio environment can cause the formation of unidirectional links. Utilization of these links and not only the bi-directional links improves the routing protocol performance.

(5)Security: A MANET routing protocol is vulnerable to many forms of attack. They are more prone to security replay transmission, do spoofing threats than other general wired networks because the network structure is not strictly defined. Also a number of nodes keep on getting added as well as deleted from the network making it very easy for a malicious node to enter a network. Then it will be relatively easy for that node to snoop on network traffic, redirect traffic and flood the entire network. Security is very important to stop any kind of disruption of the network.

(6)Power conservation: The nodes in the ad-hoc network can be laptops and thin clients such as PDA $^{\text {ee }}$ s that are limited in battery power and therefore uses some standby mode to save the power. It is therefore very important that the routing protocol has support for these sleep modes.

(7)Multiple routes: To reduce the number of reactions to topological changes and congestion multiple routes can be used. If one route becomes invalid, it is possible that another stored route could still be valid and thus saving the routing protocol from initiating another route discovery procedure.

(8)Quality of Service Support: Some sort of Quality of service is necessary to incorporate into the routing protocol. This helps to find what these networks will be used for. It could be for instance real time traffic support. It should be noted that none of the proposed protocols have all these properties, but it is necessary to remember that the protocols are still under development and are probably extended with more functionality. 


\section{CLASSIFICATION OF ROUTING PROTOCOLS IN MANET}

Protocol Classifications Depending on how protocols handle data packets from source to destination, MANET [1] routing protocols are broadly classified into three categories (Fig 1): Proactive, Reactive and Hybrid protocols.

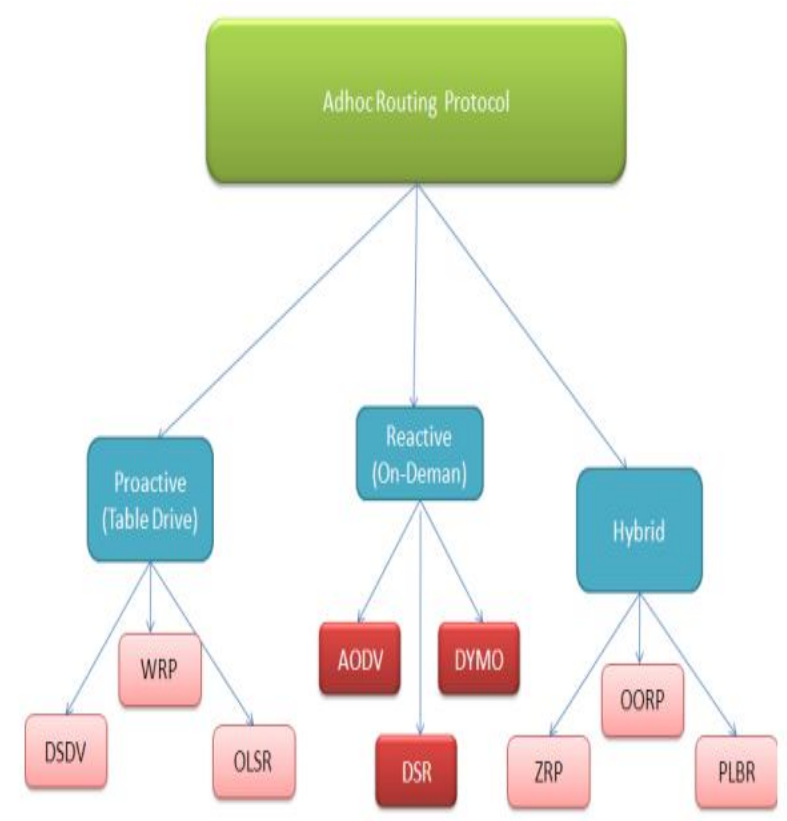

Fig1. Classification of Routing protocols in MANET

3.1.1. Proactive protocols: In proactive protocol, each node has to continuously maintain the routing table in the network. The routing information is up to date to preserve the modern view of network. Proactive protocol lowers the amount of traffic overhead because packets are forwarded only to known routers. This is inappropriate for high dynamic networks because routing table are continuously updating with change in topology, this tends in increasing the packet overhead which lower the network performance. Proactive protocol uses the shortest path protocol [12]. Proactive protocols are not suitable for large networks as they need to maintainnode entries for each and every node in the routing table of every node [13]. Some of the proactive routing protocols are Destination Sequenced Distance Vector (DSDV), Wireless Routing Protocol (WRP), hierarchical source Routing (HSR), Global state Routing (GSR).

(i)Optimized Link State Routing (OLSR):The Optimized Link State Routing Protocol (OLSR) is an IP routing protocol optimized for mobile ad-hoc networks, which can also be used on other wireless ad-hoc networks. OLSR is a proactive link-state routing protocol, which uses Hello and Topology Control (TC) messages to discover and then disseminate link state information throughout the mobile ad hoc network. Individual nodes use this topology information to compute next hop destinations for all nodes in the network using shortest hop forwarding paths.

The proactive routing protocols have the following common Disadvantages:

1. Respective amount of data for maintaining routing information.

2. Slow reaction on restructuring network and failures of individual nodes. 
Proactive routing protocols became less popular after more and more reactive routing protocols were introduced.

(ii)Destination-Sequenced Distance Vector (DSDV): Destination-Sequenced Distance-Vector Routing (DSDV) is a table-driven routing scheme for ad hoc mobile networks based on the Bellman-Ford algorithm. It was developed by C. Perkins and P. Bhagwat in 1994. The main contribution of the algorithm was to solve the routing loop problem. Each entry in the routing table contains a sequence number. If a link presents the sequence numbers are even generally, otherwise an odd number is used. The number is generated by the destination, and the emitter needs to send out the next update with this number. Routing information is distributed between nodes by sending full dumps infrequently and smaller incremental updates more frequently [6].

(iii)Wireless Routing Protocol (WRP): The Wireless Routing Protocol (WRP) is a proactive unicast routing protocol for MANETs. WRP uses an enhanced version of the distance-vector routing protocol, which uses the Bellman-Ford algorithm to calculate paths. Because of the mobile nature of the nodes within the MANET, the protocol introduces mechanisms which reduce route loops and ensure reliable message exchanges.

Table 1.Comparison of Proactive Routing Protocols

\begin{tabular}{|l|c|c|c|}
\hline \multicolumn{1}{|c|}{ Parameters } & DSDV & WRP & OLSR \\
\hline Route updates & Periodic & Periodic & Periodic \\
\hline Loop free & Yes & Yes & Yes \\
\hline $\begin{array}{l}\text { Routing } \\
\text { overhead }\end{array}$ & High & High & Low \\
\hline $\begin{array}{l}\text { Caching } \\
\text { overhead }\end{array}$ & Medium & High & High \\
\hline Throughput & Low & Low & Medium \\
\hline Routing tables & 2 & 4 & 4 \\
\hline
\end{tabular}

3.1.2. Reactive Protocol: Reactive protocol builds up routes only when required by source node. The major benefit of this protocol is that it requires minor routing information. When a communication occurs from source to destination, it includes the route discovery process. The route rest justifiable till the destination is attained or the route is no more required or expired. The route discovery happens by flooding the route request packet through the network. When reactive protocol querying for routes there is more network overhead in flooding process. Requires bandwidth only when needed. Some examples of reactive protocol are Ad-hoc On Demand Routing (ADOV), Dynamic Source Routing (DSR), and Location Aided Routing (LAR), temporally ordered Routing Algorithm (TORA).

\section{(i)AODV (The Ad hoc On Demand Distance Vector)}

The Ad hoc On Demand Distance Vector (AODV) routing algorithm is a routing protocol designed for ad hoc mobile networks. AODV is the most efficient on demand protocol used in MANET. AODV is capable of both unicast and multicast routing. AODV is an on demand algorithm, which means that it builds routes between nodes only as desired by source nodes and maintains these routes as long as they are needed by the source. Additionally, AODV forms trees which connect multicast group members. The trees are composed of the group members and the nodes, needed to connect the members. AODV uses sequence numbers to ensure the freshness of 
routes. An important feature of AODV is the maintenance of time-based states in each node: a routing entry not recently used is expired. If a route is broken the neighbors can be notified. Route discovery is based on query and reply cycles, and route information is stored in all intermediate nodes along the route in the form of route table entries. Control packets are used to accomplish above task are as follows : routing request message (RREQ) is broadcasted by a node requiring a route to another node, routing reply message (RREP) is unicast back to the source of RREQ, and route error message (RERR) is sent to notify other nodes of the loss of the link. HELLO messages are used for detecting and monitoring links to neighbors.

\section{Characteristics of AODV}

- On- demand routing with small delay.

- Link breakages in active routes efficiently repaired.

- Sequence numbers are used to make all the routes loop free.

- Use of Sequence numbers to track accuracy of information.

- Instead of the entire route it only keeps track of next hop for a route.

- HELLO messages are used periodically to track neighbors.

\section{(ii)DSR (The Dynamic Source Routing protocol)}

The Dynamic Source Routing protocol (DSR) is a simple and efficient routing protocol developed at CMU in 1996, specifically for use in multi-hop wireless ad hoc networks of mobile nodes [11]. DSR allows the network to be completely self-organizing and self-configuring, without the need for any existing network infrastructure. DSR, a reactive unicast protocol is based on source routing algorithm. In source routing, when a source node wants to send a packet, it first searches for an entry in its route cache. If the route is available, the source node includes the routing information inside the data packet before sending it. If entry is not available, the source node initiates a route discovery operation using route request (RREQ) packets. Each RREQ packet is uniquely identified by source address and unique no known as request id. On receipt of RREQ packet, an intermediary node checks its route cache. If the node doesn't have routing information for the requested destination, it appends its own address to the route record field of the route request packet. Then, the request packet is forwarded to its neighbors. A node processes route request packets only if it has not seen the packet before and its address is not presented in the route record field. If the route request packet reaches intermediate node has routing information to the destination or the destination node, a route reply packet is generated. Route reply packet is generated by the destination node, and then it comprises addresses of nodes that have been traversed by the route request packet. Otherwise, if the route reply packet is generated by intermediate node it comprises the addresses of nodes the route request packet has traversed the route in the intermediate node's route cache.

\section{Advantages}

- Guaranteed loop free routing.

- Operation in networks containing unidirectional links.

- Reduction of route discovery overheads with the use of route cache.

- Supports multi path routing.

- Does not require any periodic messages. 


\section{Limitations}

- DSR is not very effective in large networks, it performs well in static and low-mobility environments and the performance degrades rapidly with increasing mobility.

- Routing overhead is involved due to the source-routing.

- Packet size keeps on increasing with route length, because of source routing.

- DSR suffers from high route discovery latency.

\section{(iii)TORA (Temporally ordered routing protocols)}

The Temporally-Ordered Routing Algorithm (TORA)[7] is an on demand highly adaptive, efficient and scalable routing protocol. TORA is proposed for highly dynamic mobile, multi-hop wireless network that possesses the following attributes.

- Distributed execution,

- Loop-free routing,

- Multipath routing,

- Reactive or proactive route establishment and maintenance,

- Minimization of communication overhead via localization of algorithmic reaction to topological changes

TORA belong to a class of algorithms called link reversal algorithms. TORA is distributed execution, in that routers need only maintain information about adjacent routers (i.e., one-hop knowledge). Like a distance-vector routing approach, TORA maintains state on a perdestination basis. The destination-oriented nature of the routing structure in TORA supports a mix of reactive and proactive routing on a predestination basis. During reactive operation, sources initiate the establishment of routes to a given destination on- demand. This may be advantageous in dynamic networks. At the same time, selected destinations can initiate proactive operation, matching traditional table driven routing. This allows routes to be proactively maintained to destinations for which routing is repeatedly required.

\section{A. Advantages}

- Multiple route support, i.e. failure or removal of any of the nodes is quickly resolved without source intervention by switching to an alternate route.

- It's on demand routing nature creates DAG only when necessary.

- Good in network with large no of nodes. B. Limitations

- TORA protocol algorithm may also produce temporary invalid routes as in LMR.

- It depends on synchronized clocks among nodes in the ad hoc network. The dependence of this protocol on intermediate lower layers for certain type of functionality presumes that the link status sensing, neighbour discovery, in order packet delivery and address resolution are all readily available

TABLE 2: Comparison of Reactive Routing Protocols 


\begin{tabular}{|l|c|c|c|}
\hline \multicolumn{1}{|c|}{ Parameters } & AODV & DSR & TORA \\
\hline Route Creation & By source & By source & Locally \\
\hline Periodic updation & No & No & No \\
\hline $\begin{array}{l}\text { Performance } \\
\text { Metrics }\end{array}$ & Speed & Shortness & Speed \\
\hline Routing overhead & High & High & High \\
\hline Caching overhead & Low & High & Medium \\
\hline Throughput & High & Low & Low \\
\hline Multipath & No & Yes & Yes \\
\hline Route updation & Non-periodic & Non-periodic & $\begin{array}{c}\text { High } \\
\text { routing } \\
\text { overhead }\end{array}$ \\
\hline
\end{tabular}

3.1.3. Hybrid Protocol: The Hybrid protocol is the combination of both proactive routing protocol and reactive routing protocol. They have higher latency than proactive routing protocols. Some examples of Hybrid routing are Zone Routing Protocol (ZRP), Zone Based Hierarchical Link State (ZHLS). ZRP [3] divide the network into zones. The Reactive routing is used to route the packet $\mathrm{b} / \mathrm{w}$ various zones. Hybrid routing is an appropriate way for routing in large networks.

\section{i) Zone Routing Protocol (ZRP)}

ZRP [3] is suitable for wide variety of MANETs, especially for the networks with large span and diverse mobility patterns. In this protocol, each node proactively maintains routes within a local region, which is termed as routing zone. Route creation is done using a query-reply mechanism. For creating different zones in the network, a node first has to know who its neighbors are. A neighbor is defined as a node with which direct communication can be established, and that is, within one hop transmission range of a node. Neighbor discovery information is used as a basis for Intra-zone Routing Protocol (IARP), which is described in detail in [3]. Rather than blind broadcasting, ZRP uses a query control mechanism to reduce route query traffic by directing query messages outward from the query source and away from covered routing zones. A covered node is a node which belongs to the routing zone of a node that has received a route query. During the forwarding of the query packet, a node identifies whether it is coming from its neighbor or not. If yes, then it marks all of its known neighboring nodes in its same zone as covered [2]. The query is thus relayed till it reaches the destination. The destination in turn sends back a reply message via the reverse path and creates the route.

\section{CONCLUSION}

In this paper, we have survey on the taxonomy of routing protocols in mobile ad hoc networks and provided comparisons between them. The protocols are divided into three main categories: (i) source-initiated (reactive or on-demand), (ii) table-driven (pro-active), (iii) hybrid protocols. For each of these classes, we reviewed and compared several representative protocols. While there are still many challenges facing Mobile ad hoc networks related to routing and security. Each routing protocol has unique features. Based on network environments, we have to choose the suitable routing protocol. The comparison we have presented between the routing protocols indicates that the design of a secure ad hoc routing protocol constitutes a challenging research problem against the existing security solutions. We hope that the taxonomy presented in this paper will be helpful and provide researchers a platform for choosing the right protocol for their 
work. At last we have provided the overall characteristic features of all routing protocols and described which protocols may perform best in large networks.

\section{REFERENCES}

[1] C K Ton, Ad Hoc Mobile Wireless Networks, Prentice Hall Publishers, 2002.

[2] UdayachandranRamasamy," Classification of Self Organizing Hierarchical Mobile Ad hoc Network Routing Protocols - A Summary", (IJACSA) International Journal of Advanced Computer Science and Applications, . Vol. 1, No.4 October, 2010. 86

[3]Charles E.Perkins and PravinBhagwat. Highly dynamic Destination-Sequenced Distance-Vector routing (DSDV) for mobile computers. In Proceedings of the SIGCOMM '94 Conference on Communications Architectures, Protocols and Applications, pages 234-244, August 1994. A revised version of the paper is available from http://www.cs.umd.edu/projects/mcml/papers/Sigcom m94.ps.

[4] Sima," SIMULATION STUDY OF AODV\&DSR", International Journal of Computing and Business Research ISSN (Online) : 2229- 6166 Volume 2 Issue 3 September 2011.

[5] David B. Johnson, David A. Maltz and Yih-Chun Hu.The Dynamic Source Routing Protocol for Mobile Ad Hoc Networks. Internet-Draft, draft-ietfmanet- dsr-10.txt, July 2004

[6] Vincent D. Park and M. Scott Corson. Temporally-Ordered Routing Algorithm (TORA) Version 1: Functional specification. Internet-Draft, Draft-ietf-manet-tora-spec-00.txt, November 1997.

[7] Park VD, Corson MS (1997) A highly adaptive distributed routing algorithm for mobile wireless networks. Proceedings of IEEE INFOCOM 1997, Volume 3:1405-1413 Haas ZJ,

[8] Harminder S. Bindra," Performance Evaluation of Two Reactive Routing Protocols of MANET using Group Mobility Model", International Journal of Computer Science 2010; 7(3): 38-43, 2010

[9] UdayachandranRamasamy," Classification of Self -Organizing Hierarchical Mobile Ad hoc Network Routing Protocols - A Summary", journal of Advanced Computer Science and Applications,.Vol. 1, No.4 October, 2010.

[10] Yu-Chee Tseng," On Route Lifetime in Multihop Mobile Ad Hoc Networks", IEEE TRANSACTIONS ON MOBILE COMPUTI NG 1536-1233/03@2003 IEEE

[11] Nilima N. Patil et al .," Secure Routing for MANET in Adversarial Environment" International Journal of Computer Engineering In Research Trends Volume 3, Issue 4, April-2016, pp. 199-203

[12] Elizabeth M. Royer"A Review of Current Routing Protocols for Ad Hoc Mobile Wireless Networks" University of California, Santa Barbara Chai-KeongToh, Georgia Institute of Technology, IEEE Personal Communications, pp. 46-55, April 1999.

[13] Krishna Grantable, "Routing Protocols in Mobile Ad-hoc Networks", A Master' thesis in computer science, pp1- 36, 2006. 